\title{
ISOSTATIC COMPENSATION CONSIDERED AS A CAUSE OF THRUSTING ${ }^{1}$
}

\author{
BY ANDREW C. LAWSON
}

(Presented in abstract before the Society December 29, 1921)

\section{CONTENTS}

The limits of length of overthrust blocks........................... Page

The limits of movement of overthrust blocks. . . . . . . . . . . . . . . . . .

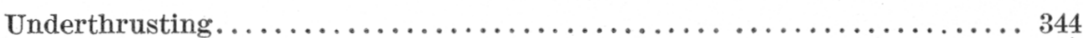

Compensatory flow and underthrusting.................. 349

\section{The Limits of Length of overthrust Blocks}

If a rupture plane of low dip exist in the earth's crust emergent at the surface, and if a stress exist in the overlying prism which tends to move it toward the emergent side, the movement is restrained by the friction of the surfaces in contact on the rupture plane. If, further, the stress

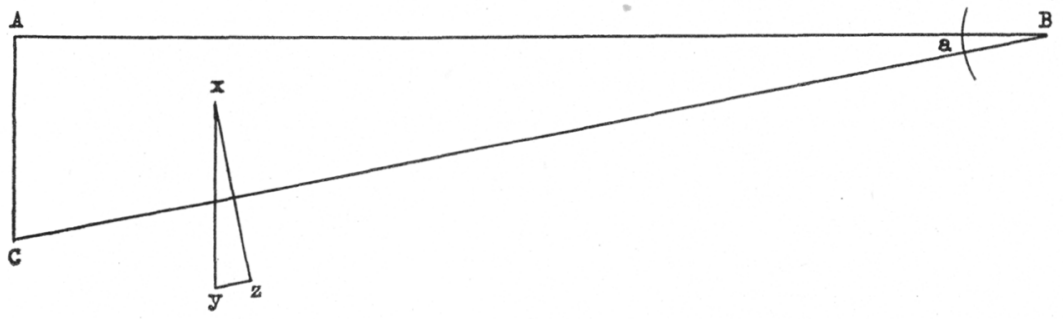

Figure 1.-Diagrammatic Representation of a Thrust Block $\mathrm{AB}$, surface of the earth; $\mathrm{BC}$, thrust plane.

be due to a pressure applied from without to the cross-section of the prism, then, in order that the prism may move, and so be thrust over the underlying rock-mass, the pressure must exceed the friction on the rupture plane. If the stress be tangential in direction and uniformly dis-

\footnotetext{
${ }^{1}$ Manuscript received by the Secretary of the Society November 14, 1921.

This paper is one of a series composing a symposium on isostasy. In the absence of the author, it was presented by H. F. Reid.
} 
tributed in the cross-section of the prism, then the limiting length of the prism which may be moved is determinable; since, when the stress necessary to overcome the frictional resistance becomes greater than the strength of the material of the prism, a new rupture will be inaugurated.

Let $\mathrm{ABC}$ be a wedge-shaped prism of the earth's crust, $\mathrm{AB}$ being the surface of the earth, and $\mathrm{BC}$ a fracture plane which has been established at the initiation of a thrust.

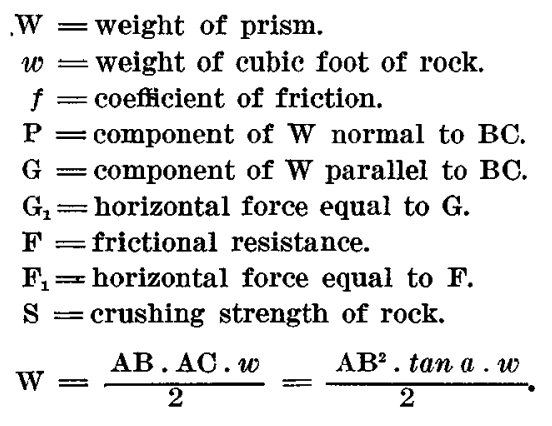

Make $x y z$ similar to $\mathrm{BCA}$ and $x y=\mathrm{W}$.

Then $\mathrm{P}=x z=x y \cos a=\mathrm{W} \cos a=\frac{\mathrm{AB}^{2} \cdot \tan a \cdot \cos a \cdot w}{2}=\frac{\mathrm{AB}^{2} \sin a \cdot w}{2}$

$$
\begin{aligned}
& \mathbf{F}=f \cdot \mathbf{P}=\frac{f \cdot \mathbf{A B}^{2} \cdot \sin a \cdot w}{\ddot{2}} \\
& \mathbf{F}_{1}=\frac{\mathbf{F}}{\cos a}=\frac{f \cdot \mathbf{A B}^{2} \cdot \sin a \cdot w}{2 \cos a}=\frac{f \cdot \mathrm{AB}^{2} \cdot \tan a \cdot w}{2} \\
& \mathrm{G}=y z=x y \sin a=\mathrm{W} \cdot \sin a=\frac{\mathrm{AB}^{2} \cdot \tan a \cdot w \sin a}{2} \\
& \mathrm{G}_{1}=\frac{\mathbf{G}}{\cos a}=\frac{\mathrm{AB}^{2} \cdot \tan a \cdot w \cdot \sin a}{2 \cos a}=\frac{\mathrm{AB}^{2} \cdot \tan ^{2} a \cdot w}{2} \\
& \mathrm{~F}_{1}+\mathrm{G}_{1}=\mathbf{A C} \cdot \mathrm{s} \\
& \text { that is, } \frac{f \cdot \mathrm{AB}^{2} \cdot \tan a \cdot w+\mathrm{AB}^{2} \tan ^{2} a \cdot w}{2}=\mathbf{A B} \cdot \tan a \cdot \mathrm{s} \\
& \mathbf{A B}=\frac{2 \mathrm{~S}}{(f+\tan a) w} .
\end{aligned}
$$

As the angle $a$ becomes larger, the expression $(f+\tan a) w$ increases, so that the value of $A B$ decreases.

That is to say, the greatest possible length of a thrust-block, measured in the direction of the thrust-movement, is for the case where the thrustplane is horizontal.

This value of $A B$ varies with the thickness of the prism in so far as the crushing strength varies with depth. But we are concerned only with the minimum value of $\mathrm{S}$, namely, that at the surface, since when the 
stress exceeds this value a new rupture will start at the surface and be propagated downward.

For any given angle $a$ which a thrust-plane makes with the horizon we arrive at a limiting value for $\mathrm{AB}$ by assuming concrete values for $\mathrm{S}, w$, and $f$ :

$w$ may be taken as .0\%5 ton per cubic foot;

$\mathrm{S}$, for the strongest rocks at the surface, may be taken as 15 tons per square inch, or 2,160 tons per square foot.

The value of $f$ is more debatable. Under laboratory conditions for the determination of engineering data, the maximum value of $f$ for rock on

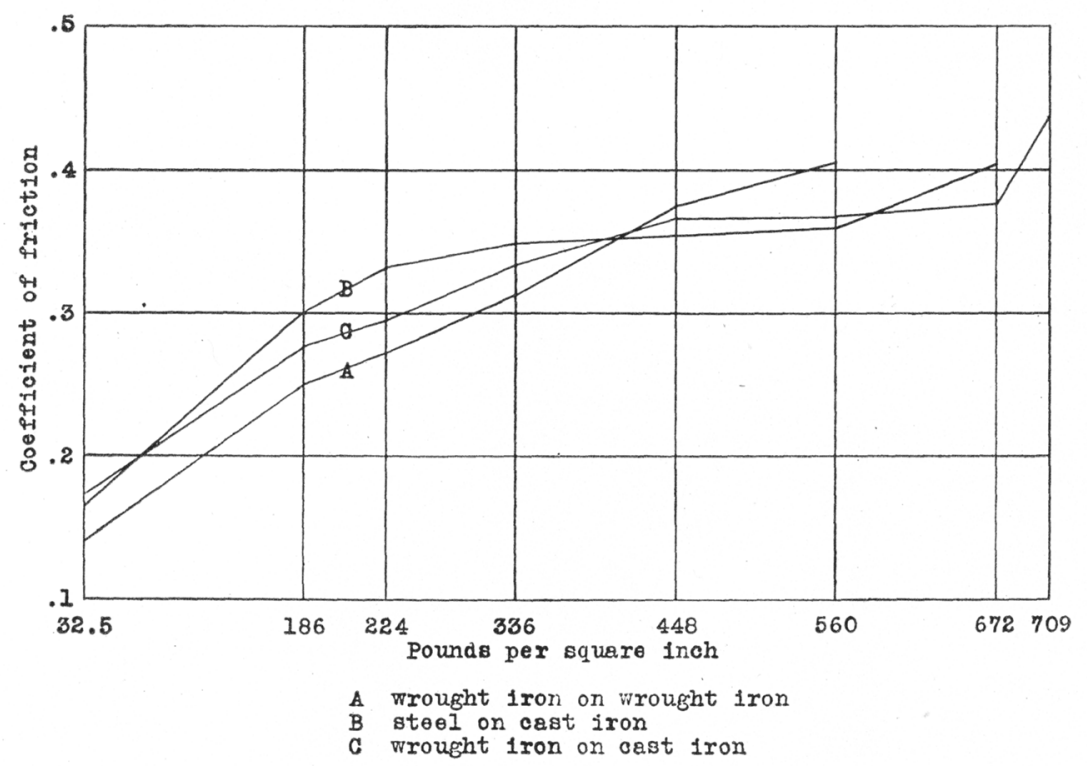

FIGURE 2,-Diagram to show the Increase in the Coefficient of Friction with increasing Pressure. (After Rennie.)

rock is about .65 . We may assume .5 as a fair figure for this quantity, as ordinarily determined; but the value of the coefficient of friction both in the laboratory and in nature varies with varying conditions. Certain experiments reported in engineering handbooks indicate that up to the limit of abrasion the coefficient of friction increases with increasing pressure.

The graph in the accompanying diagram, figure 2, constructed from results obtained by G. Rennie, ${ }^{2}$ shows the rise of the value for the coefficient of friction with increase of pressure.

'John C. Trautwine: The civil engineer's pocket-book, 1900, p. $374 b$. 
In nature the pressures are very commonly such that, when movement occurs on a rupture plane, the limit of abrasion is exceeded, as is shown by the prevalence of gouges and fault breccias. The frictional resistance to movement is not the same when the opposed surfaces are dry as when they are wet, nor is it the same when the surfaces are polished as when they are rough. I have found experimentally that when polished granite is pulled horizontally over polished granite with a bed of fine dry sand between the two surfaces, the figure obtained for the coefficient of friction is twice as great as when the polished surfaces are in direct contact; that when the sand is damp the value is two and a half times the value found when no sand is used, and that when the sand is saturated with water the value is three times as great. These results indicate that when the pressure is sufficient to develop a fault breccia the frictional resistance is thereby increased. When, however, a fault breccia is pulverized to a gouge and then saturated with water, the gouge may, perhaps, act as a lubricant. Gouge, moreover, may be developed directly by abrasion without the intervention of the condition of coarse breccia; but movement on an initial rupture is antecedent to the development of gouge, and this early movement is, therefore, not promoted by lubrication.

In the case presented I have postulated an existent rupture without qualification as to the presence or absence of a gouge lubricant and without consideration of its mode of origination. Such a rupture, however. could only occur in the earth's crust as an expression of the relief of strain, which had accumulated to the limit determined by the strength of the rocks. The stress inducing this strain may have been applied to the prism above the consequent rupture plane, in the direction toward its emergence, or to the region below that plane in the opposite direction. In the case before us consideration is limited to the first of these two possibilities. In such a case it is presumable that the stress in the superficial prism of the crust, having just exceeded the strength of the rocks at the surface and initiated a rupture there, would immediately be relatively intensified in the still unruptured region, so that the rupture would be propagated rapidly back and down until it was partially relieved in the whole of the prism affected. The residual stress would be measured by the frictional resistance on the rupture plane thus established. It is thus conceivable and probable that an intact prism might be detached from the crust on its lower side; but with the extension of the rupture plane frictional resistance would increase till it balanced the motive stress. In the development of such a rupture the amount of movement would be slight, being limited to that necessary for the first culmination of strain. This movement would be greatest at the surface and would diminish to 
zero toward the origin of stress. With this limited movement there would be small opportunity for the development of a gouge which might serve as a lubricant. Such attrition as might occur would probably result in a breccia whereby the frictional resistance would be increased.

From these considerations as well as the probability that the coefficient of friction increases with increasing pressure, it is assumed that the coefficient of friction on the rupture plane postulated is not less than that determined for similar rocks experimentally under slight pressures.

By substituting the assumed values for $\mathrm{S}, w$, and $f$ in the equation $\mathrm{AB}=\frac{2 \mathrm{~S}}{(f+\tan a) w}$, we may obtain a concrete expression for $\mathrm{AB}$ - that is, the limiting value for the length of an overthrust wedge of the crust, measured in the direction of the thrusting for any angle of thrust. Since $f$ is the doubtful factor in the equation, the doubt may be recognized by adopting a series of values for that factor.

Limiting Values for Length of overthrust Prism

$\begin{array}{rccc}a & f=.5 & f=.4 & f=.3 \\ 2^{\circ} & 20 \text { miles } & 25 \text { miles } & 32 \text { miles } \\ 5^{\circ} & 18 \text { miles } & 22 \text { miles } & 26 \text { miles } \\ 10^{\circ} & 16 \text { miles } & 19 \text { miles } & 23 \text { miles } \\ 20^{\circ} & 12 \text { miles } & 14 \text { miles } & 16 \text { miles }\end{array}$

In arriving at these values for $A B, S$ has been taken at 15 tons per square inch, or 2,160 tons per square foot, which is the maximum crushing load for the strongest rocks at the surface. Any diminution of this factor would proportionally reduce the value of $\mathrm{AB}$. It is probable that the proper value for $S$ is between the crushing load and the shearing strength, and this would be notably less than the value assumed.

\section{The Lmits of Movement of overthrust Blocks}

'The elastic rebound theory of faulting teaches that when a slip occurs on a fault, both sides of the fault move suddenly, each in an opposite direction, and that the amount of movement is limited to that necessary to relieve the elastic strain in the rocks. 'The stress which generates the strain may be persistent, so that by repetition of relief by slip the aggregate relative displacement may be large. The relief from strain at every slip may, and probably does, involve an equal amount of fling on each side of the fault; but the aggregate effect of repeated slips may be that of a mobile block moving past a passive block, the former advancing with cach slip, while the latter simply oscillates between the positions of strain 
and relief. In geological studies it is of importance to discriminate between the mobile and the passive blocks, since when we can do this we know the direction of the origin of stress. In the case of a real overthrust the upper block is the mobile side of the fault, and it would seem that the stress which is the cause of the progressive advance of the block could scarcely be applied otherwise than horizontally and fairly uniformly to its cross-section. Any other non-uniform application of the stress would probably result in a limiting value for $A B$ less than that found above.

For a real overthrust, therefore, it appears that the limiting length of the overthrust prism in the direction of the force applied is between 20 and 30 miles when the thrust-plane is nearly horizontal, and less than this if the thrust-plane is inclined. It follows from this limitation on the length of overthrust blocks that, if the stress which causes the displacement persist, relief of renewed strain can only be effected by a new

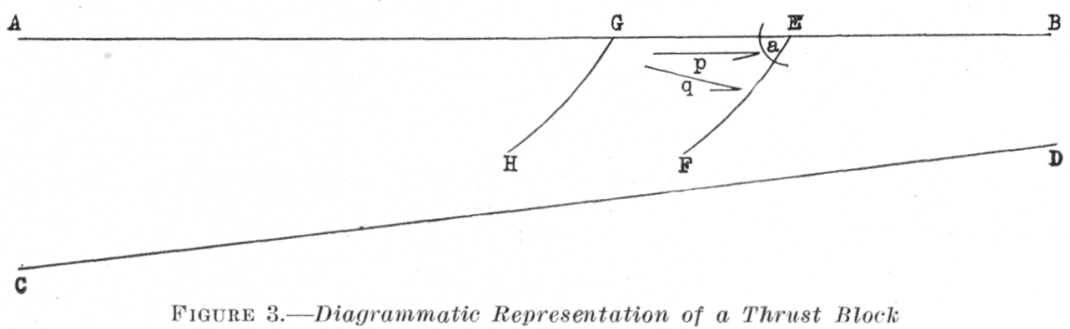

$A B$, surface of the earth; CD, thrust plane; EF and GH, successive curving thrusts due to failure of thrust block.

fault rupture situated nearer the origin of the stress. In this way, by a prolongation of the process, an imbricated structure may be produced, the fault blocks overriding each other in orderly succession like tiles on a roof.

This limitation on the length of an overthrust block, measured in the direction of the thrust, places a still more important limitation on the amount of movement by real overthrusting on a fault plane; for if it be true that 20 to 30 miles is the limiting length of the overriding block, then any displacement relative to the passive block-say, for example, one mile-must affect the whole of the block, except for slight differences in the condition of compressive strain; that is to say, the end of the block nearer the origin of stress must move approximately as much as the more remote end. But this could only happen if the ground still nearer the origin of stress were also moving, and this implies a frictional resistance to movement in excess of the strength of the rocks, as has been shown in arriving at the limiting value of $\mathrm{AB}$. It seems, therefore, mechanically 
impossible, a priori, that a single intact prism of the earth's crust could move more than a small fraction of a mile by real overthrusting as a mobile block past a passive underlying block.

In the diagram, figure 3 , let $\mathrm{AB}$ be the surface of the earth and $\mathrm{CD}$ an assumed rupture plane on which the prism ABCD tends to move to the right by a stress from the left. Let EF be the new rupture induced by a stress less than the frictional resistance on CD, but greater than the strength of rocks at the surface. The rupture EF will presumably start at the surface, where the strength is least, and be propagated downward. There will be relative displacement on EF, but this will be limited to elastic rebound and therefore will be small in amount. At first EF may be small, but if the stress be persistent the strain will, after the first relief, again accumulate to an excess of the strength of the rock and the rupture will be extended. Finally, by repetition of this process, the rup-

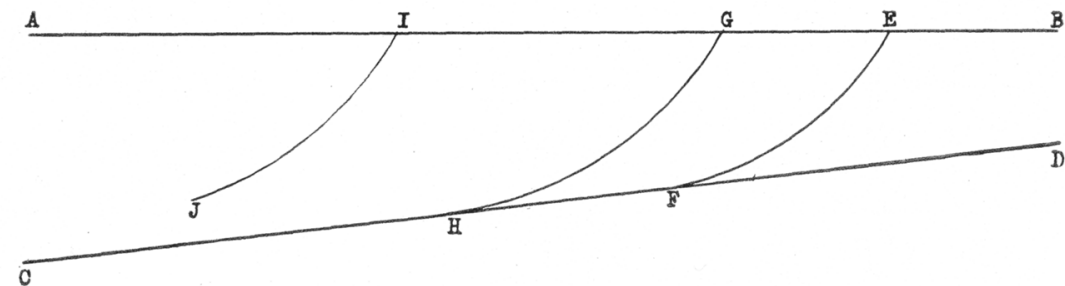

Frgure 4.-Diagrammatic Representation of a Thrust Block

AB, surface of the earth; CD, thrust plane; EF, GH, IJ, successive thrusts due to failure of thrust block.

ture EF will reach a limit, determined by the angle $a$, at which friction on this plane will also inhibit movement, and no farther extension of the crack can take place. Further accumulation of strain can then only be relieved by another rupture, as at GH, and so on.

Here two cases are possible as to the relation of the successive ruptures $\mathrm{EF}, \mathrm{GH}$, and so forth, to the original fracture CD: In accordance with engineering experience, these ruptures will make a high angle with the direction of stress - that is, with the horizontal. The horizontal distance between $\mathrm{E}$ and $\mathrm{F}$, and therefore $\mathrm{EF}$ itself, is a function of the angle $\mathrm{AEF}$, becoming less as the angle is larger. Before reaching the limit of its possible length EF (and similarly GH, and so forth), it may encounter and merge with CD, as in figure 4 ; or, if CD be too far below the surface, $\mathrm{EF}, \mathrm{GH}$, and so forth, will terminate before reaching it, as in figure 3 ; but in neither case can there be large movement on the ruptures EF, GH, and so forth. It is to be noted, however, that, in the case where CD is limited in length and is shallow, so that the new highly inclined ruptures 
merge with it, then the mobile prism becomes successively AEFC, AGHC, and so forth; and if a new rupture arise sufficiently near to C, CD may be extended down; but a limit to this extension will be reached when CD becomes too deep to be intersected by the later steep ruptures IJ, and so forth.

It appears, therefore, that in the case of real overthrust, where the lower is considered as the passive block and the upper as the mobile block, there can be no large relative displacement on any single block, owing to the fact that strain is relieved by a succession of limited ruptures and the development of an imbricated structure. It follows, of course, that the assumed lowly inclined rupture with which we started, if it exceed the limit found for $A B$, could not have been formed by real overthrusting.

In the diagrams, figures 3 and 4, the assumption is implied that the secondary ruptures EF, GH, and so forth, are curved, with concavity toward the origin of stress. The consideration underlying this assumption may be briefly stated: Prior to the inauguration of the rupture EF at the surface, the direction of stress is horizontal. If now this rupture be initiated for a depth, say, of one mile from the surface, and be inclined to the horizon, as engineering experience indicates it should be, then the angle which the new rupture plane makes with the direction of stress tends to be constant; but the moment that the first mile of rupture is opened and the strain in the upper part of the prism is thereby relieved, the direction of stress is changed from the direction indicated by the arrow $p$ to that of the arrow $q$, in figure 3 ; and, since the angle tends to remain constant, the direction of $\mathrm{EF}$, for the second mile of its extension, will change by the same angular amount. Since both the extension of $\mathrm{EF}$ and the change of direction of the stress are coincidentally progressive, EF will be curved and concave toward the origin of the stress.

\section{UNDERTHRUSTING}

We may now consider the case in which, for an assumed rupture of low dip in the earth's crust, the lower block is the mobile element and the upper the passive.

In the diagram, figure 5, let $\mathrm{AB}$ be the surface of the earth and $\mathrm{CD}$ the rupture plane. By assumption the region below CD tends to move toward the left by a stress from the right. In this case we may think of the stress either (1) as applied to the cross-section of the lower block, pushing it forward, or (2) as a gravitative stress, acting on every particle within the block, or (3) as applied to the bottom of the block by a viscous current. 
1. If the force be applied so that the block is pushed, then the latter will behave much in the same manner as the upper block did when it was considered the mobile element, except that the strength of the rock will be greater and the limits of the secondary rupture will also be greater; that is to say, that the block below CD would be broken up with the development of an imbricated structure. The imbrication of the block below CD would, however, be restrained by the load of the block above it. If this restraint were overcome, the block above CD would be correspondingly broken, probably by normal faults; but as neither the imbrication of the lower block nor the breaking of the upper block, by reason of a stress applied as a push to the cross-section of the mobile block, would produce the phenomena of large thrusting, the case will not further be considered.

2. If the force be applied to all parts of the mobile block below CD, then the frictional resistance on $\mathrm{CD}$ may be incompetent to restrain the

A

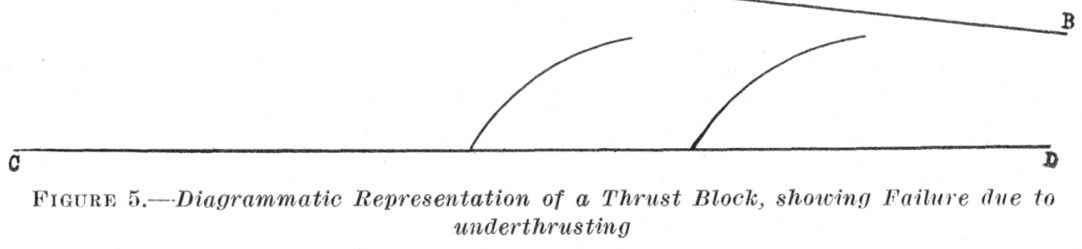

$A B$, surface of the earth; CD, thrust plane.

movement of the lower block, and the relative displacement may be indefinite in amount, without imbrication or other breaking up of the lower block.

The stress transmitted, however, to the upper block by reason of friction on CD may generate a strain in that block which will exceed the strength of the rock, and secondary ruptures will then be developed in it. This stress will not be applied to the cross-section of the upper block, but to its lower part, and will have a non-uniform distribution in the crosssection. Under these conditions the strain in the upper block near CD may exceed the strength of the rock in that region, while the strain at the surface may be less than the strength of the rocks near the surface. Thus the ruptures that relieve the strain may start at CD and be propagated upward. If this happen, which seems probable, then these ruptures, by reason of the progressive change in the direction of stress consequent on progressive relief of strain, would be concave downward or toward the 
direction of stress. The geometric relation of these curved rupture planes to $\mathrm{CD}$ is such that any movement on them, affording temporary relief of compressive strain, would develop another strain, which could only be relieved by normal faulting. These curved ruptures would be limited, both as to their extent and the amount of movement on them, by the frictional resistance on their surfaces; and this limit might be reached before they intersect the surface, or, if CD be not too deep, they might actually intersect the surface. In the latter case, or in the former after exposure by erosion, we would have an imbricated structure in the upper block, but no large displacement on the planes of imbrication. The imbricating ruptures would be distinguished from those developed in a mobile upper block by the reversal of the curvature. In the common case, in which CD is inclined, some of these secondary ruptures might reach the surface, while others, originating at greater depth, might fail to do so.

Now, since it has been shown that the mobile lower block, by gravitative stress, may move past the upper passive block to an unlimited extent, it is apparent that the same stress would have been competent to develop the rupture CD with which, for the sake of simplicity of discussion, we started; for, if we imagine a portion of the earth's crust actuated by gravitative stress so distributed that it tends to move past and under a neighboring passive block, the tendency will result in shear when the stress exceeds the strength of the rocks. Prior to rupture in the shear zone the upper passive block would fail and develop an imbricated structure; but one of the ruptures thus developed, namely, that most nearly coincident with the shear zone, or the boundary between the mobile and passive regions, would be susceptible of extension, owing to the universality of the force below this zone and the incompetence of friction to restrain its operation. This extension of the shear rupture would constitute a fault-plane on which relative displacement might take place that would be unlimited by frictional resistance. It would be an apparent overthrust but a real underthrust.

The extension of the shear rupture would relieve progressively the strain in the mobile block, and the direction of stress in the portion in advance of the rupture would, therefore, progressively change; and, on the principle that the angle which the plane of rupture makes with the direction of stress tends to be approximately constant, the direction of the rupture would correspondingly change. The latter would consequently become curved with the concavity to the right, or toward the direction of stress. This curvature of the upper surface of the mobile block indicates that as it passed the upper passive block it would do so by 
rotation on a horizontal axis deep in the earth's crust, as shown in the diagram, figure 6 , in which $\mathrm{AB}$ is the surface of the earth, $\mathrm{CD}$ the rupture, and $O$ the axis of rotation. The depth of $O$ is determinable if the curvature of $\mathrm{CD}$ is known. As displacement proceeds, the vertical component of movement at $\mathrm{D}$ is small and the horizontal component large, while the reverse is true at $\mathrm{C}$. The large vertical movement at $\mathrm{C}$ necessitates a transfer of mass by viscous flow from below the mobile block, past the lower end of the rupture $\mathrm{CD}$, to the region to the left of $\mathrm{C}$, and a consequent rise of the surface at $\mathrm{A}$. If $\mathrm{A}$ be a region of vigorous degradation, this transfer of mass from the right of $\mathrm{C}$ to the left may be a manifestation of isostatic adjustment and may in itself induce the gravitative stress in the mobile block below CD which causes it to rotate.

3. The third possible application of stress to a lower mobile block moving past an upper passive block is by the operation of a viscous current

A

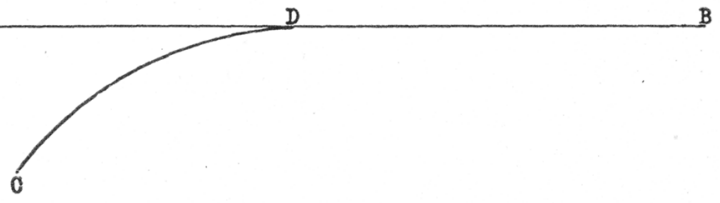

$\circ$

Figure 6.-Diagram illustrating Thrusting by Rotation

$\mathrm{AB}$, surface of the earth; CD, curve thrust; 0 , axis of rotation.

below it. Here the stress would be applied to every point of the lower surface of the block and would be non-uniformly distributed in its crosssection. In the diagram, figure $\%$, let $\mathrm{AB}$ be the surface of the earth, CD an existent lowly inclined rupture, and MN the boundary between the rigid crust and an underlying viscous region. Let the current below MN be from right to left. The strain in the prism CDNM will be torsional, since the friction on CD is counter to the stress applied to MN. If the strength of the rocks in this prism be sufficient to withstand the tendency to rupture, and the current carry the prism with it, the latter will move on CD against friction and an imbricated structure, due to failure, will be developed in ABDC. The uniform application of stress at all points on MN makes this possibility not improbable. If, however, CDNM fail, the failure would occur in its lower part first because of the greater intensity of strain in that region relatively to strength. As a result of fail- 
ure, innumerable shear ruptures would be formed and the rock would be caused to flow. This means that the boundary MN would be moved up and that the prism CDNM would finally become thin enough to insure the transmission of a stress to all points on CD sufficient to overcome friction, when the lower block would move past the upper.

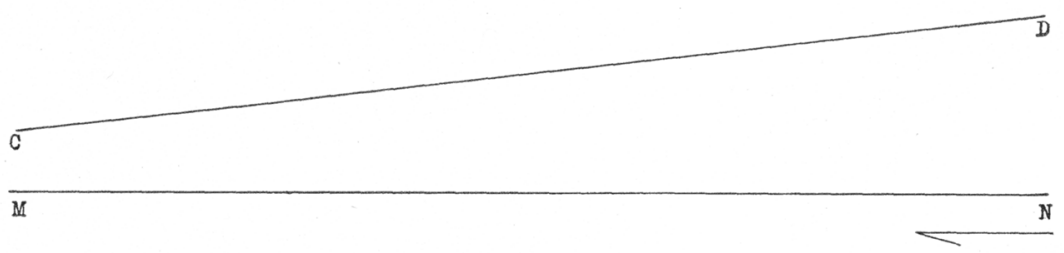

Figure 7.-Diagram illustrating Thrusting by a Current moving from Right to Left below the Crust

But if a deep viscous current be competent to cause displacement on CD against friction, it is also competent, for reasons previously advanced, to inaugurate and extend the rupture CD, and probably in this case the rupture would be started in depth and extended toward the surface, as in figure 8. With the initiation of the rupture, one of two things would happen. Either the mobile wedge would be driven under the passive

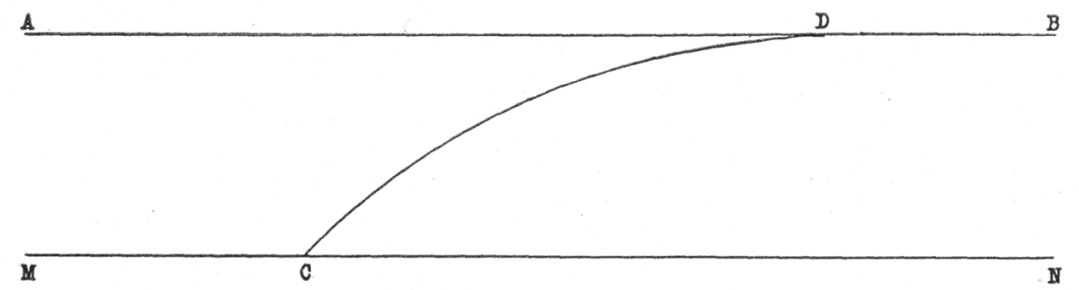

FIGURE 8.- Diagram illustrating the Initiation of a curved Thrust, D, in Depth and its Propagation toward the surface.

block, causing its uplift, or the point of the wedge would be driven downward into the viscous current and be incorporated in the latter, also causing uplift beyond the intersection of the rupture with the top of the current. Inasmuch as the rupture would be concave to the right, it seems probable that the wedge would be forced down into the current, that a rotary motion of the mobile block would develop, and that the condition of transfer of mass would be analogous to those set forth in case 2 . 


\section{Compensatory Flow and Underthrusting}

Let $\mathrm{ABC}$, figure 9, be an elevated land area in isostatic equilibrium with the area CD, the surface of which is at sealevel. Let the prism $\triangle B C E$ be transferred to $C D$ by erosion. As a result of the disturbance of equilibrium, CD is depressed to CFD, and the mass of the prism CDF is equal to that of the prism $\mathrm{ABCE}$, though the volumes may differ, owing to the less density of the newly laid sediments. The profile $\mathrm{ABC}$ is reduced and tends to assume the form EC. The profile EC is, however, never attained, owing to continuous compensation as the transfer of load proceeds. For purposes of discussion, we may assume that compensation, instead of proceeding pari passu with the transfer, begins after the latter
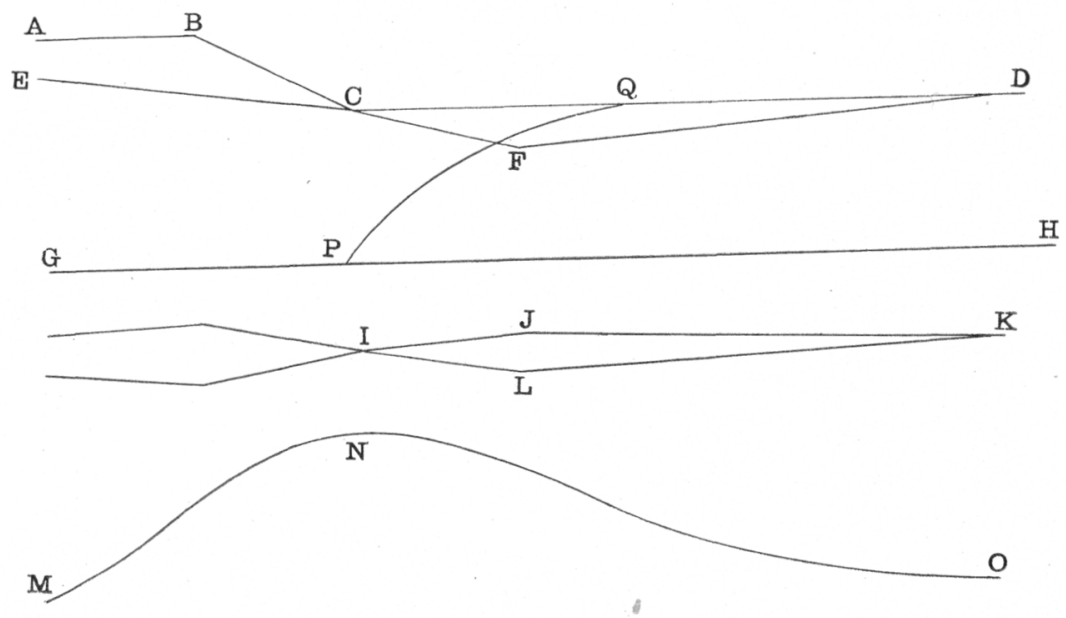

FIGURE 9.-Diagram illustrating the Development of the curved Thrust, PQ, as a Result of isostatic Compensation

has been completed, and that finally balance is restored, with no more residual than initial strain.

Compensation is effected by a deep transfer of a mass from the region under CD to the region under CE, and this mass is the same as that which has been moved from the left to the right of $\mathrm{E}$ at the surface. The deep transfer is effected by viscous flow of solid rock. The depth at which this transfer takes place is unknown, except that it probably lies within the limits of the zone of compensation, which may be taken at $\% 5$ miles. The viscosity doubtless diminishes with increasing temperature, and at some particular unknown temperature the curve of decreasing viscosity probably bends abruptly. Below the level marked by this temperature we 
may, therefore, recognize a relatively viscous condition, in contrast to a rigid condition above. Let $\mathrm{GH}$ denote the boundary between the rigid and the viscous condition. Below GH a mass, represented by the prism IJKL, ${ }^{3}$ is transferred from the right to the left of CI. If this transfer be effected in a stream of uniform thickness, the flow of which begins throughout at the same time and ends at the same time, then the relative velocity of the latter is inversely proportional to the mass passing the points at which the velocity is compared. Under this assumption the relative velocity of the stream throughout the flow is indicated by the curve MNO, which is also the curve of the square root of the momentum of the moving mass. If the transfer is effected by a stream of unequal thickness, then the curve MNO still indicates the relative momentum of the moving mass, but not the velocity.

As appears in the diagram, figure 9, the maximum mechanical effect of the viscous stream is at the point $\mathrm{I}$, or directly below $\mathrm{C}$, the border line between relief and load in the transfer of mass by erosion. Whatever, then, may be the stress applied to the crust above GH by the viscous stream, that stress (that is, drag effect) would probably have its maximum in the region under $\mathrm{C}$, and we should expect, therefore, the greatest strain and the first manifestation of underthrusting to be located to the left of CI; but, as has already been indicated, the stress would probably overtake the strength of the rocks at GH before doing so at the surface, and any rupture that occurred would be initiated at GH and be extended to the surface. Let $P Q$ be such a rupture, on which underthrusting immediately proceeds. The mobile block to the right would be thrust under the passive block to the left either by wedging or by rotation. If by wedging, then partial compensation would result, and the viscous current, the cause of the thrust-stress, would be checked. If by rotation, partial compensation would be also effected by transfer of mass from right to left, and the urgency of the viscous stream would be similarly diminished; but if by rotation, which is the more probable, there is a more rapid relief of horizontal strain near the surface than near $\mathrm{GH}$, since the horizontal component of motion on the curved rupture is large near the surface and small near GH. The strain set up in the passive block above $\mathrm{PQ}$ induced by frictional resistance on $P Q$ would be relieved by (1) relatively short thrusts, with the development of an imbricated structure in the region below.CF; (2) folding in the stratified rocks above $\mathrm{CF}$; and (3) thrust-

\footnotetext{
3 The mass IJKL is less than the mass CDF by the amount which flows out to the right of $D$ to compensate any disturbed balance in that direction, the amount which flows in from the left of $E$ by reason of disturbed balance in that direction, and an amount corresponding to any residual strain.
} 
ing of the same beds when they have become appressed and overturned. The region to the right of $\mathrm{Q}$ would remain unfolded.

The entire process would result in a typical folded belt in which the intensity of plication and thrusting is probably proportionate to amount of movement necessary to effect compensation; and this in turn is proportionate to the mass transferred by erosion from the left to the right of $\mathrm{C}$; that is, the most profoundly plicated and faulted mountains are developed in regions of heaviest sedimentation, which is a fact well known to geologists.

It should be noted that the plication and thrusting can only proceed while the deep compensatory current is flowing, thereby furnishing the necessary stress; but so long as the current flows and underthrusting is effected by rotation on $P Q$, a portion of the light rigid crust under $P Q$ is being pushed down into the heavier viscous subcrust, which, in so far as it is not removed by the current, induces elevation of the surface of the folded region by flotation. In this circumstance we have a probable explanation of the uplift of folded regions into mountain ranges. This elongation of the column under the folded belt necessitates, of course, a displacement of the viscous layer at its base, thereby inducing currents both to the right and the left of the folded region, the former eventually neutralizing the causal current of the whole complex condition of strain and so bringing the process to a close.

It is further to be noted that the loading of the region CD determines a tendency to uplift to the right of $\mathrm{D}$ as well as to the left of $\mathrm{C}$, and that underthrust movement induced by the viscous current implies a tensile strain in the neighborhood of DH. Since, however, part of the mass that moves from under CD flows to the right of $\mathrm{D}$, the maximum tension is probably located in depth between $D$ and $Q$, and this condition may explain the development of a batholithic magma in that region. 
